\title{
HR DIAGRAM OF EVOLVED STARS
}

\author{
D. SCHÖNBERNER \\ Institut für Theoretische Physik \& Stemwarte \\ Universität Kiel \\ $2300 \mathrm{Kiel}$ \\ Germany
}

ABSTRACT. A short review is presented on the various methods to construct the HR diagram for central stars of planetary nebulae (CPN).

\section{Introduction}

The localisation of central stars in a HR diagram is plagued by their very high surface temperatures and rather low apparent brightnesses. Most widely used are indirect methods that make use of nebular emission lines $\left(\mathrm{e} . \mathrm{g} . \mathrm{H}_{\mathrm{B}}\right.$ or He II 4686) to get information about the central ionizing star (cf. Harman \& Seaton, 1966). Only for about 10 years it became possible to analyze directly photospheric lines because sophisticated n-LTE atmospheric codes and more powerful telescopes became available. By plotting the stellar parameters effective temperature and surface gravity in a $T_{\text {eff }} g$ diagram, the evolutionary status can be determined distance independently upon comparison with evolutionary calculationes of post-AGB stellar models (cf. Mendez 1991, and references therein). Only a rather limited number of central stars can be studied in this rigorous way, and I will thus concentrate on the indirect methods since these are the only ones that can be used for more distant planetaries just like those in the Galactic Bulge or the Magellanic Clouds. Since the distances to these stellar systems are known, I will not discuss the problems that are connected with distance determinations.

\section{Stellar temperatures}

\subsection{Zanstramethod}

This frequently used method makes use of the fact that the planetary absorbs stellar UV photons $(\lambda<912 \AA)$ and converts them into photons with lower energy by recombination. Theory then connects the flux in selected important recombination lines to the stellar flux, allowing a determination of the stellar temperature: the Zanstratemperature $T_{x}$. For a reliable temperature determination, it is important to check whether the planetary is optically thick in the corresponding ionizing continuum, i.e. for $\lambda<912 \AA\left(\mathrm{T}_{\mathrm{z}}(\mathrm{H})\right)$ or for 
$\lambda<228 \AA\left(\mathrm{T}_{\mathrm{z}}\left(\mathrm{He}\right.\right.$ II)). Otherwise, $\mathrm{T}_{\mathrm{z}}<\mathrm{T}_{*}$ and $\mathrm{L}_{\mathrm{z}} \sim \mathrm{T}_{\mathrm{z}}^{4}<\mathrm{L}_{*}$ ( even if the distance is known!). The drastic effects of low optical depths on the loci of central stars in a HR diagram has been demonstrated by Schönberner \& Tylenda (1990). It appears that $\mathrm{T}_{\mathrm{z}}$ (He II) is the more reliable temperature, and its often noted difference to $T_{z}(H)$ can be explained by different optical depths in the corresponding ionizing continua.

If low optical depths are taken into account, useful result can still be obtained. For instance, Tylenda et al. (1991) estimated the variations of the optical depths of model planetaries along post-AGB evolutionary tracks and found for a large sample of Galactic Bulge CPN a mean mass of $0.593 \mathrm{M}_{\odot}$, with a surprisingly low scatter of only $\pm 0.025 \mathrm{M}_{\odot}$.

A variation of the Zanstramethod is that by Ambartsumyan (1932), in which the flux ratio $\mathrm{F}_{4686} / \mathrm{F}_{\mathrm{H}_{0}}$ is used to infer the temperature $\mathrm{T}_{\mathrm{A}}$ of the central source. This method works only if the planetary is optically thick in both ionizing continua. Otherwise temperatures and luminosities are overestimated (since the optical depth is always lower for wavelengths below $912 \AA$ compared to those below $228 \AA$ ). The advantage of this method is that the central star must not be known, and by this reason it has recently gained some interest (Jacoby \& Kaler, 1989; Kaler \& Jacoby, 1989).

\subsection{Energy balance method}

This method goes originally back to Stoy and uses the fact that the summed flux of all collisionally excited lines, compared to that of $H_{B}$, measures the average excess energy of the photoelectrons, which than can be converted into a stellar temperature, $T_{\mathrm{EB}}$. Again, information about the central star is not needed, and, more important, optical-depth effects cancel themselves out to a large degree. However, since most detectors have only a limited spectral coverage, one has to account for unobserved collisionally excited lines. Otherwise, $\mathrm{T}_{\mathrm{EB}}<\mathrm{L}_{*}$, and $\mathrm{L}_{\mathrm{EB}}<\mathrm{L}_{*}$ ( Henry et al., 1989; Schönberner \& Tylenda, 1990).

\section{Luminosities}

The determination of the central star's luminosity is only straightforward if its radius can be determined via its angular diameter: then $L_{*}=4 \pi \sigma R_{*} T_{*}^{4}$. Otherwise, if the star is invisible, one has to resort to the recombination theory which indicates that $\mathrm{L}_{*} \sim \mathrm{L}_{\mathrm{H}^{3}}$, the factor of proportionality $(\approx 100 \ldots . .200)$ being a function of stellar temperature and dust obsorption within the PN. Again it is important that the optical depth in the Lyman continuum is large. If this is not the case, the stellar luminosity can be greatly underestimated. (cf. Schönberner \& Tylenda, 1990). 
Another method consists of adding up all energy radiated away by recombination and collisionally excited lines, nebular continuum emission and, if necessary, by infrared emission, and correct for stellar radiation longward of $\lambda=912 \AA$. Again, a reliable stellar luminosity can only be obtained if all photons below $\lambda=912 \AA$ are absorbed.

\section{Detailed modelling}

This method is very eloborate and costly and thus not often used. The basic principle is to match the observed emission lines by a model planetary illuminated by a central source. The best match gives than $\mathrm{T}_{*}$ and $\mathrm{R}_{*}$ (distance known) and hence the stellar luminosity. Since the number of escaping UV photons is known from the models, the optical depth of the PN poses no problem. Since knowledge about the central star is not necessary, this method is also applicable to distant objects. When applied to data of sufficient quality and wavelength coverage, this method should give the most reliable results when compared to the other indirect methods discussed above. For instance, Aller et al. (1987) and Monk et al. (1987) investigated bright planetaries in the Magellanic Clouds and found their central stars to lie in a narrow strip $( \pm 0.04$ $\mathrm{M}_{\odot}$ wide!) around the $0.6 \mathrm{M}_{\odot}$ post AGB evolutionary track.

\section{References}

Aller, L.H., Keyes, C.D., Moran, S.P., Gull, T.P., Michalitsianos, A.G., Stecher, T.P., 1987, Astrophys. J. 320, 159

Ambartsumyan, V.A., 1932, Pulkovo Obs. Circ. No. 4,8

Harman, R.J., Seaton, M.J., 1966, Mon. Not. R. astr. Soc. 132, 15

Henry, R.B., Liebert, J., Boroson, T.A., 1989, Astrophys. J. 339, 872

Jacoby, G.H., Kaler, J.B., 1989, Astron. J. 98, 1662

Kaler, J.B., Jacoby, G.H., 1988, Astrophys. J. 345, 871

Méndez, R., 1991 in Stellar Atmospheres-beyond classical models, eds. Crivellari, Hubeny, Hummer (Kluwer) p. 331

Monk, D.J., Barlow, M.J., Clegg, R.E.S., 1987, IAU Symp. 131, Planetary Nebulae, p. 355

Schönberner, D., Tylenda, R., 1990, Astron. Astrophys. 234, 439

Tylenda, R., Stasinska, G., Acker, A., Stenholm, B., 1991, Astron. Astrophys. 246, 221 